Pedagogía y Saberes . $^{\circ} 52$ Universidad Pedagógica Nacional

Facultad de Educación. 2020. pp. 23-35

\title{
Voces infantiles y gestos pedagógicos en la escuela rural*
}

\section{Artículo de investigación}

\section{Child Voices and Pedagogical Gestures in Rural Schools} Vozes infantis e gestos pedagógicos na escola rural

\section{Para citar este artículo:}

Buriticá, D. y Saldarriaga, Ó. (2020). Voces infantiles y gestos pedagógicos en la escuela rural. Pedagogía y Saberes, 52, 23-35. DoI: https://doi.org/10.17227/pys.num52-10025

* Artículo resultado de la investigación en curso denominada: Entre gestos pedagógicos y voces infantiles: posibilidades para la participación y la agencia de los niños y niñas en las escuelas rurales del Tolima, realizado en el marco del Doctorado en Ciencias Sociales Niñez y Juventud de la Universidad de Manizales-Cinde.

** Docente tiempo completo Uniminuto Ibagué, docente catedrático Universidad del Tolima. Candidato a doctor en Ciencias Sociales Niñez y Juventud Cinde-Universidad de Manizales e investigador del grupo Desarrollo Integral de la Infancia de la Universidad del Tolima, e Infancias y Saberes de Uniminuto Ibagué.

Correo electrónico: danielburitica2@hotmail.com

Código ORCID: https://orcid.org/0000-0002-5032-8886

*** Doctor en Filosofía y Letras-Historia de la Universidad Católica de Lovaina. Profesor titular de la Pontificia Universidad Javeriana, y profesor Cinde-Universidad de Manizales. Miembro fundador del Grupo de Historia de la Práctica Pedagógica en Colombia (GHPP).

Correo electrónico: saldarri@javeriana.edu.co

Código oRCID: https://orcid.org/0000-0002-3661-0586 


\title{
Resumen
}

En este artículo se presenta una reflexión derivada de una investigación sobre la importancia de visibilizar las voces de los niños en el marco de las escuelas rurales, a través de la noción teórica y práctica de gesto pedagógico. En lo práctico, se propone utilizarlo como un condensador para captar y transformar prácticas pedagógicas. En lo teórico, se define como la articulación, en el oficio de maestro, de unas relaciones entre el saber pedagógico, las prácticas discursivas y no discursivas, las actitudes afectivas (amor pedagógico) y unos procedimientos técnicos de enseñanza. De este modo, se propone que el gesto pedagógico de los maestros de escuelas rurales tiene el poder de leer a los chicos en sus culturas locales, y a la vez permitir o impedir que los niños rurales den distintos sentidos a su infancia, a la particularidad social de ellos y ellas como agentes en el contexto escolar, social y cultural y como sujetos que se constituyen a sí mismos y a su realidad en relación con los otros y su entorno.

\section{Palabras clave}

gestos pedagógicos; participación; agencia; voces; escuela rural; niños y niñas

\begin{abstract}
The article presents a reflection derived from an investigation on the importance of the visibility of the children voices within the framework of the processes of participation and agency in rural schools, all this in relation to the notion of pedagogical gesture, understood as the articulation in the teacher`s work, within relationships among pedagogical practices, pedagogical knowledge, discursive and non-discursive practices, attitudes and technical teaching procedures. At the same time, it is proposed that the pedagogical gesture of rural school teachers may or may not allow rural children to give different meanings to their childhood, to the social particularity of them as agents in the social and cultural school context; as subjects who constitute themselves and who construct their reality in relation to others and their environment.
\end{abstract}

\section{Keywords}

pedagogical gestures; participation; agency; voices; rural school; children

\section{Resumo}

0 artigo apresenta uma reflexão resultado de uma pesquisa sobre a necessidade de visibilizar as vozes das crianças no marco das escolas rurais; acudindo à noção teórica e pratica do gesto pedagógico. Na pratica propõe-se seu uso como um condensador para atingir e transformar as praticas pedagógicas. No teórico define-se como a articulação, no oficio do maestro, das relações entre o saber pedagógico, as praticas discursivas e não discursivas, as atitudes afetivas (amor pedagógico), e uns procedimentos técnicos de ensinamento. Assim, propõe-se que o gesto pedagógico dos professores das escolas rurais tem o poder de compreender às crianças nas suas culturas locais, e ao mesmo tempo permitir ou impedir que as crianças rurais dêem distintos sentidos à sua infância, à particularidade social como agentes no contexto escolar, social e cultural e como sujeitos que se constituem a si mesmos e à sua realidade em relação com os outros e seu entorno.

\section{Palavras-chave}

gestos pedagógicos; participação; agência; vozes; escola rural; meninos e meninas 


\section{Introducción}

Darle más importancia a las voces de los niños es una manera de garantizar que su defensa está bien fundamentada y que la toma de decisiones se guíe por un panorama más completo de todos los temas importantes. [...], la defensa de los infantes no debe enfocarse exclusivamente en protegerlos o ayudarlos, sino que también debe tratar de entender sus puntos de vista.

Smith y Taylor (2010, p. 25).

$\mathrm{H}$ ay actualmente un interés en preguntarse por las voces de los niños, así como por la manera en que estos, en el contexto cultural, social, educativo e histórico, experimentan cotidianamente la infancia y cómo construyen su realidad y se constituyen en sujetos. De igual forma, también existe en la actualidad una tendencia a cuestionarse por la manera "como participan en la toma de decisiones de su familia, escuela y vecindario, en la manera de cuidarse, en el trabajo, el tiempo libre, o en las relaciones con sus compañeros y con los adultos" (Smith, Taylor y Gollop, 2010, p. 30). Asimismo, es evidente que en muchos casos "marcados como puros y pasivos por esencia, se otorga a los niños el derecho a la protección, pero al mismo tiempo se les niega la capacidad de actuar y la autonomía" (Giroux, 2003, p. 14), es decir, se desconoce su capacidad de agencia y participación.

De igual manera,

En la literatura académica y en las sociedades en general, los niños han sido objeto de fenómenos de invisibilización; es decir, sus voces y sus perspectivas han sido ocultadas u opacadas, por otras personas, generalmente adultos que se consideran que tienen más poder o capacidades. (Duque-Paramo, 2011, citada por Duque-Paramo, 2013, p. 100)

Por tal motivo, investigar desde y sobre las voces de los niños de escuelas rurales puede permitir la comprensión de los procesos significativos con los que reproducen lo social y apropian la cultura, y de igual manera, entender cuál es la implicación de los maestros rurales en estos asuntos, mediante un análisis desde lo que en este caso se denomina el gesto pedagógico. Este tiene que ver con la relación pedagógica maestro/alumno dentro de una gran diversidad de prácticas y discursos escolares mediante las cuales maestros y niños viven la escuela y le dan sentido, así como también con la manera en que el maestro a través de distintos gestos pedagógicos interpela (Butler,
1997), al estudiante, lo reconoce de cierta manera, visibiliza o no sus voces, lo juzga, lo acepta o rechaza, lo posibilita para que actúe de ciertas formas.

Así, es necesario comprender los gestos pedagógicos no solo en el sentido de describirlos como parte de los procesos comunicativos, sino desde los efectos que tienen en la vida escolar, las prácticas que engendran, las características que tienen, las formas de expresión y los sentidos que adquiere en las relaciones de los maestros y los niños, en el contexto de la escuela rural. Por tanto, aunque el gesto pedagógico puede estar vinculado a la comunicación no verbal, podemos ir más allá en la comprensión de este en el contexto escolar, en el sentido de que "permite ser develado e interpretado a la luz de sentimientos y actitudes" (Ospina, 2008), es decir, lo que el maestro expresa en su ejercicio de enseñanza, como lo hace, pero también su actitud frente a este ejercicio. De igual manera, como se expone en la figura 1, estos gestos pedagógicos parecen estar relacionados con la formación del maestro, con sus posturas epistemológicas y teóricas, con su ejercicio docente, con sus experiencias de vida y su discurso y actitud para desarrollar su labor pedagógica. Es decir, en cada maestro finalmente se configura un(os) gesto(s) pedagógicos(s) como resultado de la relación de todos estos componentes, el cual aflora en el contexto escolar cuando ejerce su práctica pedagógica.

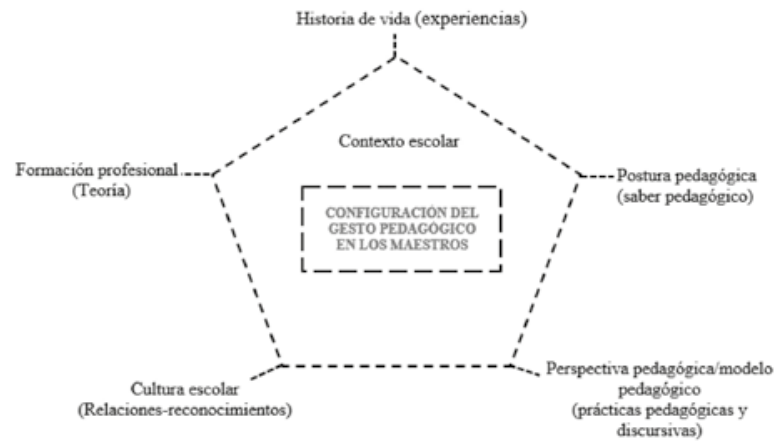

Figura 1. Configuración del gesto pedagógico en los maestros

Fuente: elaboración propia.

Finalmente, proponemos que el gesto pedagógico tiene un rol importante en las tareas de orientar mejor el acto educativo, para entender las vivencias de los niños en las escuelas y la manera en que estas adquieren sentido para ellos y se convierten en espacios de realización de la vida cotidiana, de sus intereses, sus realidades y su constitución como 
sujetos. Es necesario que el gesto pedagógico del maestro visibilice sus voces, posibilite su participación y agencia, y nos abra a leer sus concepciones sobre la vida escolar y cotidiana, sobre los maestros y sus propios aprendizajes.

\section{Las voces de los niños y niñas rurales como participación y agencia}

\section{Son finalmente los niños y las niñas quienes recrean su mundo. \\ Díaz (2010, p. 1154).}

Para comprender los asuntos de participación y agencia en los niños rurales es pertinente ubicarse epistemológicamente desde la antropología y la sociología de la infancia. Con la primera, para tener en cuenta la perspectiva de los niños, lo que hablan, lo que nos dicen (Calderón, 2015); comprender que los niños y niñas son sujetos con visiones, criterios, experiencias, significaciones propias sobre la vida y la cultura por la cual transitan (Chacón, 2015). Así, en las investigaciones sobre infancia, es primordial incluir las visiones de los niños, para entenderlas desde sus propias voces y no por los juicios y prejuicios construidos por los adultos (Chacón, 2015). Es el punto de vista emic. Un interesante trabajo en esta perspectiva es el de Ruiz Castiblanco (2013), el cual plantea una inquietante pregunta: ¿A qué edad se dejar de ser niño y se pasa a ser joven en el campo? Ella responde que en la región que estudia (cerca de Ibagué), a los 10 años un niño ya entra a hacer tareas de adulto (p. 6). Pero habrá que ver si esto es generalizable a otras regiones. Desde la segunda perspectiva, los niños son entendidos como actores y agentes sociales activos y sujetos de derecho que construyen sus propias realidades y se constituyen a partir de estas; por tanto, las investigaciones deben enfocarse directamente en ellos, sus condiciones de vida, actividades, relaciones, conocimiento y experiencias (Calderón 2015). Es el punto de vista etic.

De este modo, con la sociología se teoriza sobre la infancia, y los niños se presentan como seres sociales con capacidad de agencia; desde la antropología, se busca interactuar con los niños a través de metodologías que permitan visibilizar sus voces y comprender sus perspectivas sobre la vida y poder dar cuenta de eso.

En ese sentido, es evidente que las voces de los niños siguen, en muchos casos, ausentes o invisibilizadas, tal es el caso en el contexto educativo sobre todo en el sector rural, pues, aunque sean participantes primordiales en los procesos educativos en las escuelas, al final termina el adulto (docente y directivos) tomando decisiones y controlando lo que estos pueden hacer y decir, como y cuándo hacerlo. Las voces (participación y agenciamiento) de los niños en las escuelas terminan siendo potestad de los maestros, y según sea su gesto pedagógico, los posibilita o empodera (o no) para expresarlas y ejercerlas.

Los niños de los contextos rurales en la actualidad tienen diversas formas de vivir sus infancias. Y en todo caso, son actores y agentes que influyen en la organización social de sus familias, vereda y escuela, y a la vez son influenciados por sus demás familiares, vecinos, y en el caso de la escuela por sus compañeros y maestros. En ese sentido, los niños realizan una contribución importante para ayudarnos a comprender la naturaleza de la infancia y a solucionar los problemas que afectan sus vidas (Smith, Taylor y Gallop, 2010). Esto tiene que ver también con el hecho de que en cada contexto ellos despliegan diversas formas de vivir sus infancias (Pavez, 2012). Sin embargo, los niños rurales no han tenido garantías sociales, políticas y culturales reales que les garanticen una buena calidad de vida. El sujeto niño rural generalmente ha sido narrado y contado por otro, visto a través del otro y representado a través de los rasgos interpretados por otro. En el contexto escolar rural generalmente sus voces no han tenido posibilidad de expresarse, por tanto, se han desconocido sus formas de ver el mundo, sus culturas, sus concepciones y sus maneras de constituirse en sujetos, de participar y de construir sus realidades.

La tarea entonces será comprender las relaciones de los maestros y los niños, mediadas por el gesto pedagógico. Entendemos este, en modo hipotético, como las formas corporales, simbólicas, y las actitudes y discursos con que el maestro ejerce su práctica educativa; da lugar a los niños rurales en su calidad de sujetos, agentes y actores que habitualmente están relegados al lugar de sujetos inmaduros, incapaces e, incluso, irrelevantes.

Por eso, es importante saber si los maestros, en este caso los rurales, reconocen a los niños como agentes y actores con capacidad de reconfigurar y resignificar sus realidades sociales y culturales en su contexto. Y, en el mismo sentido, si su gesto pedagógico garantiza y estimula la libertad de expresión de los niños, su creatividad y capacidad de tener un juicio sobre el mundo que los rodea. Es importante "hacer visibles a los niños a través de la palabra, demostrar que son sujetos sociales y que sus voces son una manera de interpretar la realidad y de leer 
el mundo" (Cuervo y Rincón, 2009, p. 68), sobre todo porque, el gesto pedagógico involucra asuntos relacionados con la cultura, y un alto porcentaje de escuelas rurales están atendidas por maestros que provienen de la zona urbana, o que han trabajado en el contexto escolar urbano. En este sentido, el maestro tendrá un gesto pedagógico (y es natural) unido a su propia cultura y no a la del niño o la niña de la zona rural, "esta apertura para comprender la infancia en lo rural, dejando de lado la mirada urbanocéntrica y hegemonizante, presenta grandes dificultades y desafíos" (Ávila y Vargas, 2017, p. 228). De esa manera, será importante que el gesto pedagógico respete estas dinámicas culturales propias de cada contexto rural, teniendo en cuenta que los niños van construyendo su realidad según su experiencia escolar. De ahí la importancia de escucharlos sobre las maneras como conciben la escuela, en aras de reconocer sus voces como posibilidad para mejorar los procesos educativos y de que las instituciones educativas se conviertan en espacios en los cuales la alegría, la creatividad y la pasión por el conocimiento formen parte de la cotidianidad (Cifuentes Garzón, 2015).

Por su parte, San Fabián-Morato (2008) plantea una reflexión en torno a la participación de los estudiantes en las escuelas y a la pregunta "¿Existen alumnos en los centros o solo expedientes académicos y cuerpos disciplinados?". Frente a esto, afirma que los estudiantes son víctimas de un reducido estatus e incluso que son tratados como discapacitados sociales, lo que podría afectar la práctica de sus derechos en las escuelas. En ese sentido, lo que se espera es que se tenga en cuenta lo que dicen y piensan, ya que esto puede "ayudarnos a mejorar la enseñanza, a tejer la convivencia y a dotar de sentido su estancia en la escuela. Necesitamos revisar nuestra concepción y manera de relacionarnos con los menores, tratarlos como sujetos activos, entrar en diálogo con ellos" (p. 27).

Por otra parte, Duque-Páramo (2013) señala que generalmente, las políticas y programas de educación dirigidos a los niños "presuponen que ellas y ellos son menores de edad, seres dependientes, predominantemente vulnerables, receptores pasivos de las acciones de asistencia y protección definidas por otros, que generalmente son adultos con ideas normativas y naturalizadas de la infancia" (p. 81). Se debe, por tanto, asumir que "las niñas y los niños son al mismo tiempo sujetos abiertos, agentes y actores sociales" (p. 81).

En ese sentido, visibilizar las voces de los niños en las escuelas rurales por parte de los maestros es una tarea primordial en la educación, en la idea de que ellos necesitan de espacios formativos que los ayuden a configurarse como sujetos con capacidad de agencia, reflexión y transformación.

\section{Entre voces y gestos}

Cada voz individual está conformada por la historia cultural particular y la experiencia anterior de su propietario. La voz, entonces, sugiere los medios que los estudiantes tienen a su disposición para hacerse "oír" y definirse como participantes

activos en el mundo. Exhibir una voz individual significa, citando a Bajtin, "redecir un texto en las propias palabras"..

McLaren (2005, p. 325).

La educación es ética, es una relación de respuesta al otro, y el educador es alguien apasionado por la palabra, por la transmisión de la palabra, por la acogida y la hospitalidad, por la donación. El educador es, en primer lugar, el que transmite la palabra dicha, la palabra del pasado, de la tradición, a un recién llegado, pero no para que este la repita, sino para que la renueve, la vuelva a decir de otro modo, la convierta en "palabra viva". Pero, en segundo lugar, el educador también es aquel que recoge la pala-

bra del otro, la nueva palabra, la del recién llegado. El educador escucha la palabra del otro y él

mismo desde ella, se transforma y se renueva.

$$
\text { Mèlich (2012, p. 50). }
$$

Así como todo campo de investigación, el de la escuela y "el de la infancia está[n] compuesto[s] por enfoques, análisis, estudios y conceptos; por la práctica que incluye un conjunto de acciones, programas y políticas; y finalmente, por una amplia gama de actores intervinientes" (Bustelo, 2005, p. 254). Para este caso, será el asunto de la visibilización/invisibilización de las voces de los niños en las escuelas rurales lo que se pone en juego. Sabiendo que los niños, en general, necesitan posibilidades para mejorar su participación y construir lo social en sus territorios, ellos, en sus discursos y acciones, en sus decires y haceres, construyen su realidad (Berger y Luckmann, 2003), y se constituyen como sujetos con capacidad de agencia (Pavez, 2012), en cuanto participantes activos en la construcción de su vida cotidiana (Smith y Taylor, 2010). Y "como actores sociales que participan en la construcción y determinación de sus propias vidas, de quienes les rodean y de las sociedades en que viven" (Pavez, 2012, p. 94). En la escuela esto es importante porque entre otras cosas, genera "nuevas maneras de relacionarnos, sobre las que podríamos 
ampliar nuestro conocimiento y decisiones" (Díaz, 2010). En ese sentido, los maestros rurales deben saber que los niños "no son objetos pasivos en las estructuras sociales y en los procesos económicos o políticos, sino sujetos activos en la construcción y determinación de sus propias vidas" (Prout y James, 1990, citados por Sosenski, 2016, p. 44).

Del mismo modo, es necesario comprender desde sus voces las concepciones que tienen sobre la escuela, los maestros, su territorio y su cultura; esto, permite gestionar mejor el acto educativo, configura un gesto pedagógico en los maestros que ayuda a reconocerlos con capacidad de hacer y decir, pues, "mediante la acción y el discurso, los hombres muestran quienes son, revelan activamente su única y personal identidad y hacen su aparición en el mundo humano" (Arendt, 2005, p. 208).

Dar voz a los niños es permitir que afloren sus capacidades y expresen sus concepciones sobre la vida, saber cómo configuran y resignifican sus realidades sociales y culturales. Sobre todo, sabiendo que, en la escuela, así como en

[...] los discursos oficiales, los medios masivos de comunicación destinados a la niñez y hasta las artes gráficas y visuales han sido manufacturados por un mundo de adultos [y yo agrego, urbanos] que, en su mayoría, han buscado determinar las experiencias de los niños. (Sosenski y Albarrán, 2012, p. 11)

Generalmente "las personas adultas han privilegiado lo que piensan importante de la protección del niño o niña, y han descuidado, voluntariamente o no, garantizar y estimular su libertad de expresión" (Gómez-Mendoza y Alzate-Piedrahita, 2013, p. 84).

Entre tanto, un gesto pedagógico en los maestros rurales que visibilice sus voces puede ayudar a entender esas nuevas infancias rurales que se configuran en la actualidad porque "cuando se indaga a los niños y las niñas se advierten concepciones y reflexiones sobre la infancia que develan su representación de lo social" (García y Gallego, 2011, p. 19). Esto es necesario para el mejoramiento de las prácticas educativas rurales, en aras de que "la escuela en cuanto institución social y democrática pueda contribuir eficazmente en la formación de nuevos ciudadanos que aporten al desarrollo político, económico y social del país, tarea en la cual los docentes tienen una función trascendental" (Cifuentes, 2015, p. 165). De igual manera, para re-conocer y valorar sus puntos de vista, pensamientos, sueños, problemáticas, necesidades e intereses frente a las diversas situaciones que se dan en el espacio educativo y en la vida cotidiana rural. También para "analizar sus propias experiencias, así como a iluminar los procesos por medio de los cuales esas experiencias fueron producidas, legitimadas o negadas" (McLaren, 2005, p. 321).

Sobre el término voz del alumnado, Susinos (2012) afirma que son "todas aquellas iniciativas que emprenden las escuelas y que están dirigidas a aumentar el protagonismo del alumnado en la toma de decisiones sobre el diseño, la gestión y la evaluación de cualquier aspecto de la vida escolar" (p. 16).

Por otro lado, McLaren (2005) al referirse a la voz de los alumnos, afirma:

[...] los estudiantes no pueden aprender "con provecho" a menos que los maestros desarrollen una comprensión de las diferentes formas en las que sus percepciones e identidades son constituidas. Los maestros necesitan comprender cómo las experiencias producidas en los diferentes dominios de la vida diaria producen a su vez las distintas voces que los estudiantes emplean para dar significado a sus mundos y, por consiguiente, a su existencia en la sociedad. (p. 322)

Nuevamente McLaren (2005) retomando lo que plantea Giroux sobre la voz, señala que esta

[...] Se refiere al conjunto de significados multifacéticos y articulados con los cuales los estudiantes y los maestros se enfrentan activamente en diálogo uno con otro. La voz es un concepto pedagógico importante porque alerta a los maestros del hecho de que todo discurso está situado históricamente y mediado culturalmente, y que deriva parte de su significado de la interacción con los demás. Aunque el término voz puede referirse a un discurso internalizado, privado, tal discurso no puede ser comprendido sin situarlo en un universo de significados compartidos, esto es, en los símbolos, narrativas y prácticas sociales de la comunidad de nuestra cultura en la que el diálogo está tomando lugar. El término voz se refiere a la gramática cultural y al conocimiento que los individuos utilizan para interpretar y articular la experiencia. La voz individual debe entenderse dentro de su especificidad cultural e histórica. Cómo los estudiantes, los maestros y los demás se definen a sí mismos y nombran su experiencia es central en la preocupación pedagógica porque ayuda a los educadores a comprender cómo es producido, legitimado y deslegitimado el significado en el salón de clase. (p. 325)

En tal sentido, la visibilización de la voz de los niños en las escuelas rurales es además una posibilidad de entender cuáles son las prácticas educativas que se están privilegiando en estos contextos, el impacto en la realidad de la vida cotidiana, entendiendo que esta "se reafirma continuamente en la 
interacción de los individuos con los otros" (Berger y Luckmann, 2003) y la cultura rural de ellos, en el sentido de que "poder hablar de sí, de la experiencia cotidiana y la trama familiar, de situaciones y conflictos en los distintos ámbitos de pertenencia, es una ayuda invalorable para la creación colectiva y el aprendizaje" (Arfuch, 2016, p. 240).

Así como también para:

Hacer visibles a los sujetos ante las colectividades, pues en el ejercicio relacional se comienzan a desplegar una serie de habilidades para escuchar, reflexionar, comunicarse respetuosamente, valorar lo que otros dicen, asumir turnos, expresar pensamientos, propiciando esto el encuentro de muchas voces que favorecerían la interacción con el mundo desde una postura crítica que contribuiría a su vez con la democratización de la sociedad. (Cuervo y Rincón, 2009, p. 71)

Visibilizar las voces de los niños en las escuelas rurales es respetar el mismo lenguaje como "la capacidad intrínseca de expresividad vocal que posee el organismo humano" (Berger y Luckmann, 2003). Pero, sobre todo, es la posibilidad de conocer la realidad de su vida cotidiana, en cuanto esta "es vida con el lenguaje que comparto con mis semejantes y por medio de él. Por lo tanto, la comprensión del lenguaje es esencial para cualquier comprensión de la realidad de la vida cotidiana" (p. 53). Cada voz de los niños está permeada por su cultura; por ende, las voces representan "los medios que los estudiantes tienen a su disposición para hacerse 'oír' y definirse como participantes activos en el mundo" (McLaren, 2005).

Ahora bien, los gestos cumplen un papel fundamental para la interacción en las aulas de clase, así como la relación que, según la comunicación no verbal, establecen los docentes y sus estudiantes (Ospina, 2008). Según ella:

El gesto como texto, [...], nos dice qué hay detrás de una mirada, de una sonrisa, de un acercamiento. Nos permite leer, develar qué subyace en la interacción, en la comunicación no verbal del docente con sus estudiantes. Por ello, el gesto como texto vincula al docente [...] en interacción con sus estudiantes. Se producen gestos que en la interacción cobran vida y significado. El docente lee e interpreta ese gesto, ese texto; a su vez, el estudiante lee e interpreta ese texto, esos gestos de su maestro, en interacción pedagógica. (p. 56)

De esta manera:

[...] cobra importancia la propuesta de un corpus gestual que contenga gestos importantes para la interacción pedagógica. [...] Gestos que puedan develarse, transcribirse, analizarse, para hallar los sentidos que subyacen en ellos. Dar cuenta de un corpus gestual es develar la interacción entre el docente y sus estudiantes a partir de sus gestos, y dar cuenta de cómo se estructura, organiza y desarrolla la interacción comunicativa en la clase. (pp. 202-203)

Igualmente, la autora argumenta que

[...] los gestos invitan a los estudiantes a participar de la clase. Mediante los gestos se crean espacios interesantes para los estudiantes que incitan a intervenir en la clase. Los sumerge en el tema tratado. El docente crea interés a partir de su rostro, de su mirada, de su sonrisa; incita a la participación de sus estudiantes. De esta manera, los gestos narran con movimientos del cuerpo, las historias, las explicaciones, los contenidos de las asignaturas analizadas. Para los estudiantes, el gesto de su docente es fundamental, pues, a partir de él, del gesto del docente, el estudiante tiene acceso a los conocimientos. (p. 214)

Los gestos tienen gran influencia en los procesos de enseñanza y aprendizaje, en el sentido de que por medio de los gestos de los docentes rurales pueden generar un "ambiente de participación y agrado para los estudiantes que leen y develan sentidos en la comunicación no verbal de sus docentes" (p. 216).

En una segunda mirada, el gesto pedagógico puede ser entendido como un gesto mínimo (Ribetto, 2014); es decir,

Un acontecimiento que irrumpe y provoca experiencia. El gesto, su presencia, viene antes... antes de cualquier reforma en las leyes, de cualquier propuesta didáctica, de cualquier adaptación de la currícula, de cualquier proyecto político pedagógico. El gesto antecede estos movimientos: es una composición y un pasaje entre todos ellos. (p. 8)

La autora propone el concepto de gestualidad mínima, retomando una idea de Carlos Skliar (2011), quien propone leerlo como un agenciamiento que permite pensar el educar como una relación de alteridad posible. Algo como

[...] entender los efectos que se crean a partir de esta tensión: prácticas, experiencias, currículos, espacios, tiempos... Gestos encarnados en las relaciones entre los sujetos que viven las escuelas posibles... Gestualidad mínima que interpela "sobre el lenguaje en que formulamos lo educativo [...] sobre los modos en que se produce lo educativo [...] abriendo la posibilidad hacia una cierta forma de pensar sobre "eso que pasa", "eso que nos pasa" en la educación a diario. (Skliar, 2011, p. 260, citado por Ribetto, 2014, p. 3) 
Manghi-Haquin (2016) presenta la categoría vínculo pedagógico como un elemento primordial para desarrollar el aprendizaje curricular y la formación de los estudiantes, y expone la necesidad de que los docentes se vinculen con sus estudiantes y construyan una relación pedagógica. "Dicha relación se lleva a cabo no solo mediante las palabras que se dicen (recursos lingüísticos) sino a través de otros recursos comunicativos como miradas, posturas corporales y gestos típicos de la interacción cara a cara" (p. 11). Este cara a cara entre docentes y alumnos nos acerca a la noción de gesto pedagógico. Por su parte, Prieto (2006, citado por Manghi-Haquin, 2016) señala que "los profesores somos seres de comunicación, tenemos la necesidad permanente de interactuar con otros, de relacionarnos con nuestros aprendices, de exponernos recíprocamente a nuestras miradas, voces y gestos, lo que supera los contenidos curriculares" (p. 12).

Por su parte Castillo (2015) presenta las categorías corporeidad y gesto educativo haciendo referencia a un territorio en donde el cuerpo y las significaciones permiten nuevas posibilidades para la educación. "Un tipo de corporeidad entendida como un sentido sensible y el gesto educativo como una declaración orgánica que presenta al cuerpo-pensamiento en toda su novedad" (p. 121). Según este autor, "la importancia del gesto corporal se presenta como ademán simbólico que cimienta la construcción de un espacio cultural y educativo lleno de significaciones" (p. 126); en cuanto propone que "desde una mirada antropológica y simbólica podemos encontrar bases para construir una mirada integradora del cuerpo y el gesto en favor de la educación" (p. 128). El gesto pedagógico en este sentido también podría incidir en los sentimientos que sienten o construyen los niños sobre el territorio de la escuela rural, es decir, un tipo de topofilia - sentido de pertenencia a un territorio- (Tuan, 2007), generada a partir de los gestos pedagógicos de los maestros de estos contextos.

Por otro lado, los gestos pedagógicos surgen en las prácticas escolares y las prácticas discursivas que se dan en el contexto escolar, relacionadas con las formas de reconocer, dirigir, enseñar, presentar, exigir, evaluar, reconocer, castigar, etc. Se podría decir que estos están relacionados, por un lado, con el mostrar pedagógico (Prange, 2002, citado por Runge 2019), que se refiere a lo que se muestra cuando se enseña algo, es decir; siempre en todo acto educativo, en toda práctica escolar, se muestra algo como condición inicial de todo proceso de enseñanza y de aprendizaje, pues, "sin mostrar no hay educación, en toda parte donde se educa también se muestra algo" (Prange, 2002, citado por Runge, 2019). Asimismo, la actitud o las actitudes con que el maestro ejerce las prácticas pedagógicas y discursivas, de esta manera los gestos pedagógicos son acción educativa pero también actitud o formas y maneras de enseñar o de expresar la acción educativa, en síntesis, es lo que se enseña (muestra) y cómo se enseña (actitud).

Siguiendo a Runge (2019), diríamos que así como en el mostrar pedagógico, en los gestos pedagógicos hay tres dinámicas que los constituyen: a) de direccionalidad (el gesto está diciendo algo a alguien o a un grupo): como actos comunicativos, interpelan constantemente a los alumnos, reforzando ciertas etiquetas sobre ellos que en las prácticas escolares los docentes van asignando; formas en las que se remiten a los estudiantes para felicitarlos, regañarlos, llamar a lista, revisar tareas, etc.; b) de reconocimiento (los gestos ven de ciertas maneras, tienen formas de expresión que reconocen al otro como sujeto): las maneras como se perciben maestros/estudiantes en su diferencia y en su rol, los gestos pedagógicos visibilizan o no al otro, lo posibilitan o no para que actúe de ciertas formas, lo somete; c) de subjetivación (los gestos configuran ciertos tipos de sujetos y subjetividades): cómo alguien se constituye en sujeto, cómo se ve y lo ven los demás en el marco de los gestos pedagógicos que encarnan prácticas escolares y discursivas de los maestros.

En la figura 2 se presenta el esquema sobre los gestos pedagógicos, construido a partir de las comprensiones teóricas y empíricas expuestas en las páginas precedentes.

CONTEXTO ESCOLAR

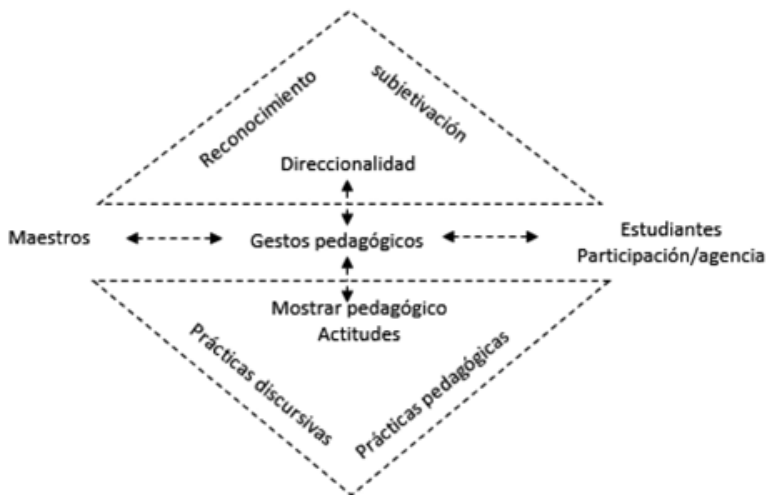

Figura 2. Esquema gestos

pedagógicos de los maestros

Fuente: elaboración propia.

En una tercera mirada, tenemos que recoger las advertencias que hacen autores respecto de la ilusión de describir el gesto pedagógico como una prolongación transparente y neutral de las buenas intenciones 
pedagógicas. Por una parte, somos advertidos, como investigadores, sobre los riesgos epistemológicos y éticos de tomar como objetos a los sujetos/niños: el artículo "Dar casa a las voces infantiles, reflexiones desde la historia" (Sosenski, 2016), plantea una fuerte discusión en torno a la utilización de las voces de los niños en las investigaciones, afirmando que utilizamos las voces de los niños mayoritariamente para elaborar textos académicos, informes, proyectos o estrategias para intentar construir soluciones a sus problemáticas específicas. Sin embargo, en la búsqueda por rescatar las voces de los niños de la marginalidad en la que se hallan ubicadas, como escritores de los textos terminamos por apropiarnos de sus palabras, y así las controlamos, editamos y hasta censuramos. En ese sentido, las relaciones entre el gesto pedagógico y el discurso pedagógico, pueden ser también contradictorias y ambivalentes.

En el artículo "Los gestos: interacción visible en las aulas de clase de la Educación Superior" (Ospina y González, 2009), las autoras argumentan sobre la importancia del gesto en la interacción pedagógica de los docentes con sus estudiantes en la educación superior y cómo el uso de la palabra articulada en la transmisión de contenidos académicos va escindiendo las miradas, los rostros, las sonrisas, las cercanías, que se manifiestan en las interacciones pedagógicas y son importantes para la comunicación de los conocimientos. Esta es una observación muy potente, pues alerta sobre la duplicidad de códigos que pueden coexistir entre el gesto y la palabra de los maestros, y es un fenómeno clave a tener en cuenta en la investigación.

Finalmente, se plantean estas tensiones en el gesto pedagógico como tensiones constitutivas del gesto pedagógico.

\section{Según Saldarriaga (2019), un gesto}

[...] no es un mero ademán lanzado al aire ni una repetición mecánica de movimientos, sino un movimiento o una serie de actos físicos, técnicos, que a la vez movilizan o emiten lenguaje, comunican con símbolos, manifiestan emociones, articulando relaciones sociales. Un ademán o cadena de ademanes dirigidos que se identifica por transmitir, ya una finalidad técnica, ya un significado o concepto, o ya una vibración afectiva, en un juego de planos relacionales. Pero hay un punto crucial a señalar: si concebimos "el gesto" como algo mucho más complejo que la ejecución física de una idea, hay que abordarlo más bien como un compuesto de técnicas (acciones funcionales) y de lenguajes (simbolismo, emoción o saber), que vinculan relaciones sociales, relaciones entre sujetos situados socialmente, culturalmente. Lo gestual nos plantea el problema de la coexistencia, tensional y hasta contradictoria, de una heterogeneidad de funciones y sentidos; la convergencia de códigos plurivalentes entre sujetos localizados en roles o "posiciones de sujeto". (p. 7)

Estas advertencias deben ser parte vital del análisis de los juegos de visibilización e invisibilización de las voces de los niños, y en particular de los niños rurales.

\section{La escuela rural (escuela nueva) como escenario para la participación y agencia de los niños}

La infancia rural, al igual que otras infancias, no se adhieren a definiciones unívocas, ya que "están sujetas a un proceso de constante lucha y negociación tanto en el ámbito público (medios de comunicación, política social, ámbito académico) como en el privado" (Sánchez, 2004, p. 150). Por lo que los niños rurales necesitan posibilidades para mejorar su participación y construir lo social en sus territorios. Ellos, en sus discursos y acciones, en sus decires y haceres van construyendo su realidad y se van constituyendo como sujetos con capacidad de agencia. No obstante, cuando se habla de infancia rural, al igual que otras infancias, como la urbana, la indígena, etc., se sabe que se trata de una concepción que, generalmente, proviene del adultocentrismo; desde esta mirada se determina qué es ser niño y lo que debe hacer un niño, generando muchas veces "interpretaciones de infancia rurales que limitan su comprensión real como sujetos que se configuran en un tejido de lo simbólico, cultural, económico particular mediado por el escenario biofísico" (Ávila y Vargas, 2017, p. 267). Flor Edilma Osorio, una destacada estudiosa de la ruralidad, y de los jóvenes rurales, confirma estas aseveraciones

La soberbia y el poder del conocimiento usualmente localizados en las ciudades, han configurado una serie de imaginarios y representaciones con respecto a lo rural y a quienes allí habitan, que deben ser reelaborados y actualizados con miras a construir unas relaciones horizontales y equitativas entre sociedades rurales y urbanas. Pero además, es necesario reconocer el protagonismo de los propios moradores rurales, con todas sus tensiones, dilemas e intereses, como los tiene cualquier sociedad. (2014, p. 18) 
Además, esta investigadora proporciona un cuadro de interpretación para detectar las situaciones identitarias posibles de hallar en estos niños rurales. Esta es su tipología, muy sugestiva:

Las identidades rurales reclaman ser leídas como identidades plurales, multidimensionales y dinámicas. Así por ejemplo, identificamos algunas formas identitarias rurales en construcción, que dan cuenta de su diversidad. Encontramos la identidad rural vivida cotidianamente, gozada y sufrida como parte de su pertenencia permanente a ese lugar, inclusive por generaciones. También está la identidad rural añorada, que se vive con las migraciones, en donde lo rural se vive como dimensión espacial de la identidad, aún cuando no se habite. Está la identidad rural buscada, en donde situamos a los neorrurales y a aquellos que buscan lo rural por una razón funcional de comodidad y bienestar, habitando allí como primera o segunda residencia, pero no sintiéndose parte, esquivando e inclusive menospreciando la sociedad rural. Está también la identidad rural asignada, imputada según comportamientos, actitudes que son identificadas como rurales (usualmente con menosprecio) frente a los modismos, la forma de expresarse o vestirse, entre otros y que como lo señala Di Méo y Buleón (2005) son muestra de estigmatización. Finalmente, encontramos una identidad rural vergonzante en donde los estigmas hacen mella y que Wacquant ha señalado como una estigmatización territorial, para el caso de las ciudades, y que es muy pertinente para el caso rural, estigmatización de la cual se puede escapar o disimular fácilmente cambiando de lugar, emigrando, como ha sucedido históricamente. (Osorio, 2014, p. 6)

Por tal motivo, lo que está en juego aquí, es identificar cómo se ubican estos niños en esas identidades a través de las relaciones de poder generacionales en donde domina lo que piensa y dice el adulto, desde sus diferentes lugares de agenciamiento, en este caso desde la escuela rural y a través del gesto pedagógico.

En el contexto de la escuela nueva, siguiendo a Villar (1995), se puede evidenciar cómo en este modelo el maestro se entendía como un continuo recreador preparado para la adaptación constante de las estrategias y un agente activo en el desarrollo del programa. Sin embargo, afirma que, debido a la expansión del programa al total de escuelas rurales del país, se presentó un enfriamiento de un modelo que buscaba ser replicado mecánicamente y que hacía del maestro un adoptador de estrategias más que un adaptador. Esto, en la actualidad, todavía representa un reto para los maestros que trabajan en este modelo: por un lado, tener que enseñar todas las áreas a todos los grados a partir de guías y textos, viéndose reducido a la sola aplicación instrumental y mecánica del modelo, un asunto basado en el "conductismo y la tecnología educativa" (Gómez, 1995), por otro, lograr una articulación de su gesto pedagógico con la cultura de estos contextos y no con la lógica de la escuela urbana, lo que implica que el maestro construya su práctica pedagógica en armonía con las dinámicas de los contextos rurales en su diversidad cultural, social y económica.

Sin embargo, Colbert (1999) presenta la escuela nueva como un buen ejemplo de una innovación local que se convirtió en política nacional colombiana, que además sirvió de inspiración a muchos otros países del mundo. Según la autora, este sistema proporciona sobre todo un aprendizaje activo, una relación más estrecha entre la escuela y la comunidad, y un mecanismo de promoción flexible adaptado al estilo de vida del niño campesino. Así las cosas, se constituye en un modelo ideal para garantizar la participación y agencia de los niños y niñas en los contextos rurales, pues entre otras cosas, permite, según la autora, la promoción flexible; es decir que los estudiantes puedan avanzar de un curso a otro según su propio ritmo de aprendizaje. Además, los niños pueden abandonar temporalmente la escuela para ayudar a sus padres en las actividades agrícolas (cosechas), en caso de alguna enfermedad o en cualquier otra situación válida, sin correr el riesgo de no poder retornar y continuar con sus estudios. Es toda una apuesta por:

[...] desformalizar la escuela, volviéndola flexible, relevante, útil, creativa, amena, sensible a la inteligencia y al pensamiento, respetuosa de la diversidad, atenta a las necesidades de los niños, receptiva a las necesidades de los maestros, abierta a la participación de los padres de familia y la comunidad, responsable por sus resultados frente a la sociedad. (Torres, 1992, p. 11)

Lo anterior indica que este modelo ofrece desde su estructura la posibilidad para la participación de los niños; su garantía va a depender entonces del gesto pedagógico de los maestros, y en todo caso "es pedagógicamente como mejor se puede iluminar la relación formativa entre el maestro, las guías, los rincones de aprendizaje, la biblioteca, el gobierno escolar y la interacción con la comunidad" (Flórez, 1995, p. 218) y la participación efectiva de los niños y niñas.

No obstante, en un análisis más reciente, Forero (2013) afirma:

La escuela rural comparte con la escuela en general el hecho de ser establecida para socializar conocimientos, pero en materia de gestión hay profundas 
diferencias cuyas implicaciones llevan a señalar que la calidad educativa allí generada no es suficiente para optimizar las condiciones de vida de las comunidades rurales, lo cual significa que el objetivo para lo cual se diseñaron no se logra, mientras no se propongan cambios sustanciales en términos de gestión y rol del docente rural de aula multigrado. (p. 7)

Igualmente, plantea que en las escuelas rurales la intervención del docente tiene una influencia fundamental en la calidad de la educación que se imparte, y donde además es específico su quehacer para orientar, planificar y organizar las condiciones referentes a las actividades pedagógicas (Forero, 2013), y por supuesto para la participación de los niños. En consecuencia, el modelo educativo flexible Escuela Nueva exige del maestro prácticas diversas, innovadoras y dinamizadoras de las acciones que desde la escuela se adelantan, así, sus acciones reiteran la necesidad de preservar elementos particulares de las comunidades (Pineda de Cuadros, 2014). De otro lado, la discusión en torno la Escuela Nueva está en que los docentes deben mejorar sus prácticas pedagógicas, actualizarse en didáctica, preparar mejor las clases, ser más dinámicos y afectuosos con los niños (Jiménez, 2016). Pero también mejorar en generar reflexiones propositivas y críticas sobre los gestos pedagógicos de los maestros rurales, la visibilización o invisibilización de las voces de los niños, y la relación maestro/alumno/cultura rural.

\section{Conclusiones}

El anterior panorama plantea importantes retos para el campo investigativo en cuanto a la educación de los niños de los contextos rurales que en la actualidad tienen diversas formas de vivir sus infancias. Y en todo caso, son actores y agentes que influyen en la organización social de sus familias, vereda y escuela, y a la vez son influenciados por sus demás familiares, vecinos, compañeros y maestros que forman parte de un medio cultural. Igualmente, la Escuela Nueva tiene sus propias características que la pueden configurar en un escenario propicio para la participación de ellos y, en ese sentido, los niños pueden realizar una contribución importante para ayudarnos a comprender la naturaleza de la infancia y a solucionar los problemas que afectan sus vidas (Smith y Taylor, 2010). Esto tiene que ver también con el hecho de que en cada contexto ellos despliegan diversas formas de vivir sus infancias (Pavez, 2012). Por ende, hay una tarea importante en el contexto de la educación rural que en gran parte requiere del compromiso y el gesto pedagógico de los maestros que están allí.
El estudio del gesto pedagógico como concepto relacionado con la escuela, la labor y la formación docente, es relativamente nuevo en nuestro contexto, aquí se intentará construir un concepto complejo, tensional, que ayude a comprender la manera en que este se configura en los maestros, a partir de las prácticas pedagógicas, discursivas y las actitudes, en relación con la visibilización o invisibilización de las voces de los niños rurales, su agencia y participación. ¿Cómo construir nuevas formas pedagógicas que hagan que la escuela sepa leer, escuchar y ver a las culturas rurales y a las infancias concretas, localizadas en ellas?

Estudiar el gesto pedagógico y la relación con los procesos de participación y agencia de los niños y niñas en el contexto de la educación rural es un tarea importante, primero, porque este implica a la vez una actitud dialógica y otra simbólica o no verbal del maestro y del niño, es decir, el gesto pedagógico no es solo lo que se expresa en un lenguaje no verbal, sino también el sentido de lo que se dice y hace en relación con los gestos, y segundo, porque este puede dar cuenta de la práctica del maestro, la participación de los niños, su reconocimiento y su inclusión en lo educativo, social, político y cultural de su territorio, y por tanto, su visibilización o invisibilización como un sujeto que participa, negocia, interviene, enseña y aprende. Y finalmente, habrá que asumir que los gestos pedagógicos no se pueden comprender desde una teoría del gesto, pues "no hay una teoría de la interpretación de los gestos" (Flusser, 1994), sino a partir de la observación y análisis (en gran medida etnográfico) de la realización del acto de enseñar en prácticas escolares concretas (clases, actividades escolares, etc.), y por ende, con actores concretos (los maestros) que al ejercerlas y ponerlas en el escenario escolar (actitudes) muestran y activan la polivalencia de los gestos pedagógicos.

\section{Referencias}

Arendt, H. (2005). La condición humana. Barcelona: Paidós.

Arfuch, L. (2016). Subjetividad, memoria y narrativas: Una reflexión teórica y política en el campo de la educación. Magis, Revista Internacional de Investigación en Educación, 9(18), 227-244. DoI: https://doi.org/10.11144/ Javeriana.m9-18.smnr

Ávila, L. y Vargas, J. (2017). Visiones de la niñez y la ruralidad en las políticas públicas para la infancia de contextos rurales durante los últimos 80 años. En C. Córdoba y A. Acosta (coords.), Las infancias múltiples (pp. 227271). 1a. ed. Bogotá: Centro Internacional de Educación y Desarrollo Humano (Cinde). 
Berger, P. y Luckmann, T. (2003). La construcción social de la realidad. Buenos Aires: Amorrortu.

Bustelo, E. (2005). Infancia en Indefensión. Salud Colectiva, 1(3), 253-284.

Butler, J. (1997). Lenguaje, poder e identidad. Madrid: Síntesis.

Calderón, D. (2015). Los niños como sujetos sociales. Notas sobre la antropología de la infancia. Nueva Antropología, XXXVIII(82), 125-140.

Castillo, R. (2015). Corporeidad y gesto educativo: repensando la escena en perspectiva situada. Infancia, Educación y Aprendizaje (IEYA), 1(1), 110-129.

Chacón, J. (2015). Antropología e infancia. Reflexiones sobre los sujetos y los objetos. Cuicuilco, 22(64), 133-153.

Cifuentes, J. (2015). Una mirada a la escuela desde las voces de unos estudiantes del sector rural. Revista Virtual Universidad Católica del Norte, 45, 159-172.

Colbert, V. (1999). Mejorando el acceso y la calidad de la educación para el sector rural pobre. El caso de la Escuela Nueva en Colombia. Revista Iberoamericana de Educación, 20, 107-135.

Cuervo, C. y Rincón, C. (2009). La voz de los niños: un pasaporte para explorar la oralidad en el aula. Bogotá: Pontificia Universidad Javeriana.

Díaz, S. (2010). Participar como niña o niño en el mundo social. Revista Latinoamericana de Ciencias Sociales, Niñez y Juventud, 8(2), 1149-1157.

Duque-Páramo, M. (2013). Investigación con niños sobre sus voces y experiencias. Intencionalidades, conceptos, aspectos éticos y métodos. En P. Lozano y S. Castellanos (ed.), Salud colectiva. Perspectivas teóricas y metodológicas (pp. 99-131). Bogotá. Editorial Pontificia Universidad Javeriana.

Flórez, R. (1995). La dimensión pedagógica: formación y escuela nueva en Colombia. Revistas Educación y Pedagogía, 14, 197-219.

Flusser V. (1994). Los gestos. Fenomenología y comunicación. Barcelona: Herder.

Forero, I. (2013). El rol del docente en la gestión educativa de las escuelas rurales multigrado, (tesis de maestría), Universidad Pedagógica Nacional.

García, G., y Gallego, T. (2011). Una concepción abierta e interdisciplinar de la infancia. Revista Latinoamericana de Ciencias Sociales, Niñez y Juventud, 9(2), 17-25.

Giroux, H. (2003). La inocencia robada. Juventud, multinacionales y política cultural. Madrid: Ediciones Morata.
Gómez-Mendoza, M. y Alzate-Piedrahíta, V. (2013). La infancia contemporánea. Revista Latinoamericana de Ciencias Sociales, Niñez y Juventud, 12(121), 77-89. DoI: https://doi.org/10.11600/1692715x.1213040513

Gómez, V. (1995). Una visión crítica sobre la Escuela Nueva de Colombia. Revista Educación y Pedagogía, 7(1415), 280-306. Recuperado de http://aprendeenlinea. udea.edu.co/revistas/index.php/revistaeyp/article/ view/5592/5014

Manghi-Haquin, D. (2016). Vínculo pedagógico en contextos escolares: más allá de las palabras y del currículo Vínculo pedagógico en contextos escolares: más allá de las palabras y del currículo. Boletín Científico Sapiens Research, 6(2), 11-19. Recuperado de http://www. sapiensresearch.org/

McLaren, P. (2005). La vida en las escuelas. Buenos Aires: Siglo XXI.

Mèlich, J. (2012). Filosofía de la finitud. Madrid: Editorial Herder.

Osorio, F. (2014). Identidades rurales en perspectiva territorial: dinámicas cambiantes en tiempos de crisis. Recuperado de https://www.academia.edu/8308669/ Identidades_rurales_en_perspectiva_territorial_ din\%C3\%A1micas_cambiantes_en_tiempos_de_crisis

Ospina, T. (2008). Interacción pedagógica del gesto del docente en la educación superior: una perspectiva didáctica. Medellín: Universidad de Antioquia.

Ospina, T. y González, A. (2009). Los gestos: interacción visible en las aulas de clase de la Educación Superior Teresita. Uni-pluri/versidad, 9(1), 1-10.

Pavez, I. (2012). Sociología de la infancia: las niñas y los niños comoactoressociales. Revista de Sociología,27(27),81-102. DoI: https://doi.org/10.5354/0719-529X.2012.27479

Piedrahíta, M. (2016). Escuela nueva prácticas pedagógicas y gestión escolar: un estudio de caso único.

Pineda de Cuadros, N. (2014). Reflexiones sobre la labor docente en escuelas rurales que implementan la metodología escuela nueva. Quaestiones Disputatae: Temas en Debate, 7(15).

Ribetto, A. (2014). Gestos mínimos y pedagogía de las diferencias. Polis, Revista de la Universidad Bolivariana, 13(37), 191-202.

Ruiz, C. (2013). Los jóvenes rurales desde su propia voz, las veredas El Retiro Resbalón y la Plata el Brillante, corregimiento de Villarestrepo del municipio de Ibagué, departamento del Tolima. (Tesis para optar por el título de maestría). Bogotá: Pontificia Universidad 
Javeriana. Facultad de Estudios Ambientales y Rurales. Recuperado de https://repository.javeriana.edu.co/ handle/10554/12397

Runge, A. (2019). Analítica pedagógica de la subjetivación y del reconocimiento: Los aportes de Michel Foucault, Judith Butlery Norbert Ricken para pensar las interacciones educativas y el mostrar pedagógico. Documento de trabajo. Línea de Investigación Educación y Pedagogía Doctorado en Ciencias Sociales Niñez y Juventud. Universidad de Manizales-Cinde.

Saldarriaga, O. (2019). Notas para una arqueología del gesto pedagógico. Documento de trabajo. Línea de Investigación Educación y Pedagogía Doctorado en Ciencias Sociales Niñez y Juventud. Universidad de Manizales-Cinde.

Sánchez, C. (2004). La construcción social de la infancia: apuntes desde la sociología. Témpora: Revista de Historia y Sociología de la Educación, (7), 149-168.

San Fabián-Morato, J. (2008). La voz de los estudiantes en los centros escolares. ¿Hay alguien ahí? OGE, 5, 27-32.

Smith, B., Taylor, J. y Gallop, M. (coords.) (2010). Escuchemos a los niños. México: Fondo de Cultura Económica.

Sosenski, S. (2016). Dar casa a las voces infantiles, reflexiones desde la historia. Revista Latinoamericana de Ciencias Sociales, Niñez y Juventud, 14(1), 43-52. DoI: https://doi.org/10.11600/1692715x.1411250315
Sosenski, S. y Albarrán, E. (2012). Introducción. En Nuevas miradas a la historia de la infancia en América Latina: entre prácticas y representaciones (pp. 7-21). México. Recuperado de http://www.historicas.unam. $\mathrm{mx} /$ publicaciones/publicadigital/libros/miradas/ mirada001.pdf

Susinos, T. (septiembre-diciembre de 2012). Las posibilidades de la voz del alumnado para el cambio y la mejora educativa. Revista de Educación, 359, 16-23.

Torres, R. (1992). Alternativas dentro de la educación formal: el programa" Escuela Nueva" de Colombia. Perspectivas: Revista trimestral de educación comparada, (4), 549-558.

Tuan, Y. (2007). Topofilia: un estudio de las percepciones, actitudes y valores sobre el entorno. Barcelona: Melusina.

Villar, R. (1995). El programa Escuela Nueva en Colombia. Revista Educación y Pedagogía, 7(14-15), 357382. Recuperado de http://aprendeenlinea.udea. edu.co/revistas/index.php/revistaeyp/article/ view/5596/5018 


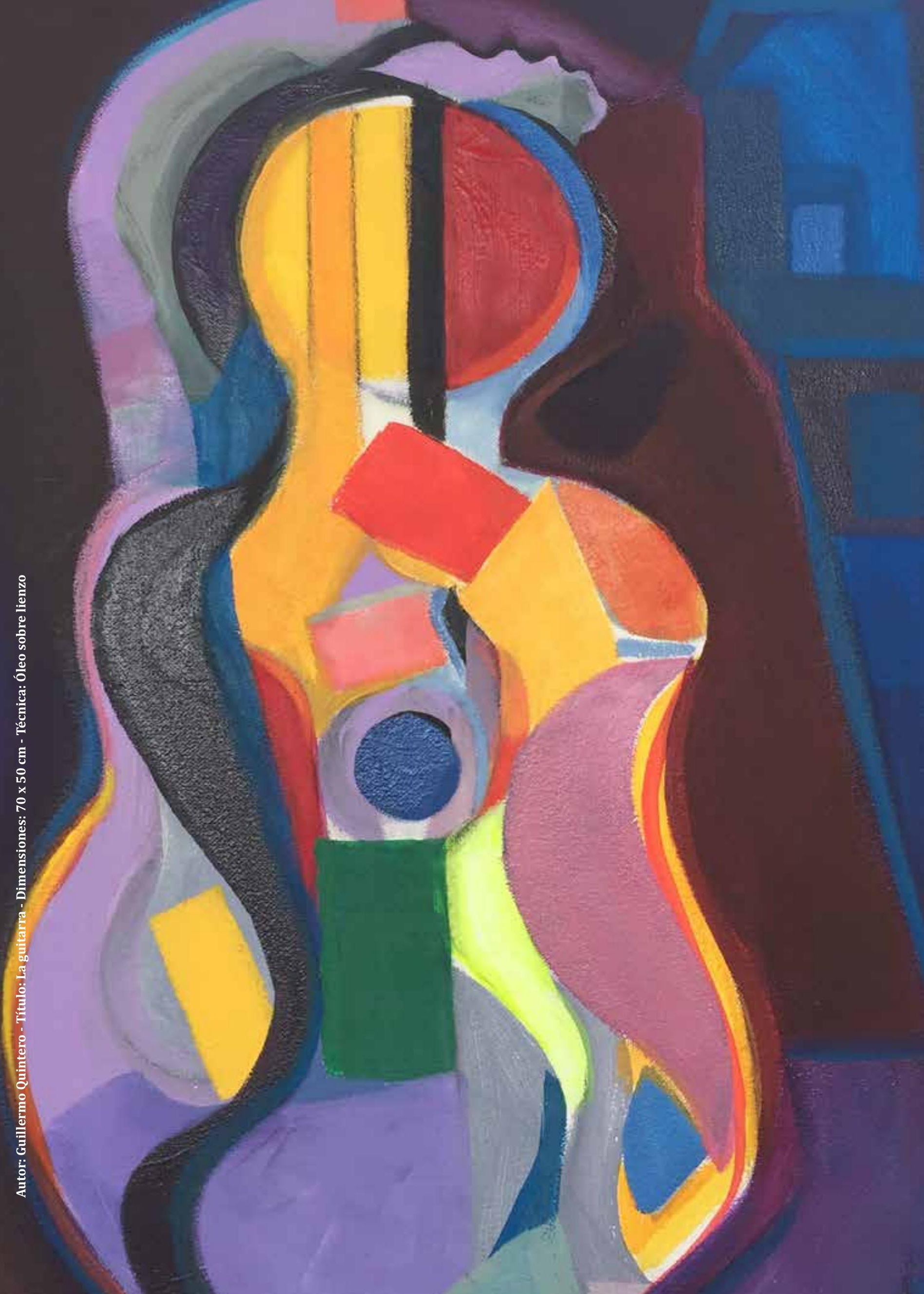

